\title{
Mobile Magnetic \\ Nanocatalysts for \\ Bioorthogonal Targeted \\ Cancer Therapy
}

\author{
Marcus Hoop,* Ana Sofia Ribeiro, Daniel Rösch, \\ Philipp Weinand, Nuno Mendes, Fajer Mushtaq, \\ Xiang-Zhong Chen, Yang Shen, Carlos Franco \\ Pujante, Josep Puigmartí-Luis, Joana Paredes, \\ Bradley J. Nelson,* Ana Paula Pêgo,* and Salvador \\ Pané*
}

M. Hoop, D. Rösch, P. Weinand, F. Mushtaq, Dr. X.-Z. Chen, Prof. B. J. Nelson, Dr. S. Pané Institute of Robotics and Intelligent Systems (IRIS) ETH Zurich Tannenstrasse 3, CH-80g2 Zurich, Switzerland

E-mail:mhoop@ethz.ch; bnelson@ethz.ch; vidalp@ethz.ch; Dr. A. S. Ribeiro,Dr. J. Paredes, N. Mendes IPATIMUP - Institute of Molecular Pathology and Immunology of the University of Porto, Rua Júlio Amaral de Carvalho, 45. 4200-135 Porto, Portugal

Dr. A. S. Ribeiro, Dr. J. Paredes, N. Mendes, Prof. A. P. Pêgo

$i_{3} S$ - Institute for Investigation and Innovation in Health, University of Porto, Portugal. Rua Alfredo Allen 208, 4200-135 Porto, Portugal

Dr. Y. Shen

Institute of Food, Nutrition and Health, ETH Zurich, Schmelzbergstrasse 7, CH-8092 Zurich, Switzerland Dr. J. Puigmartí-Luis, Dr. C. F. Pujante

Institute of Chemical and Bioengineering, ETH Zürich, Vladimir Prelog Weg 1, 8093 Zurich, Switzerland Prof. A. P. Pêgo

INEB - Instituto de Engenharia Biomédica, Rua Alfredo Allen 208, 4200-135 Porto, Portugal

E-mail:apego@ineb.up.pt

Prof. A. P. Pêgo

Instituto de Ciências Biomédicas Abel Salazar (ICBAS), Universidade do Porto, Rua de Jorge Viterbo

Ferreira 228, 4050-313 Porto, Portugal

Prof. A. P. Pêgo

Faculdade de Engenharia da Universidade do Porto, R. Dr. Roberto Frias

4200-465 Porto, Portugal

Originally published in Hoop, M., Ribeiro, A.S., Rosch, D., Weinand, P., Mendes, N., Mushtaq, F., Chen, X.-Z., Shen, Y., Pujante, C.F., Puigmartí-Luis, J., Paredes, J., Nelson, B.J., Pêgo A.P., Pané, S. "Mobile Magnetic Nanocatalysts for Bioorthogonal Targeted Cancer Therapy" Advanced Functional Materials 2018, 28(25),1705920. DOI: 10.1002/adfm. 201705920 
"This is the peer reviewed version of the following article: Hoop, M., Ribeiro, A.S., Rosch, D., Weinand, P., Mendes, N., Mushtaq, F., Chen, X.-Z., Shen, Y., Pujante, C.F., Puigmartí-Luis, J., Paredes, J., Nelson, B.J., Pêgo A.P., Pané, S. "Mobile Magnetic Nanocatalysts for Bioorthogonal Targeted Cancer Therapy" Advanced Functional Materials 2018, 28(25),1705920, which has been published in final form at https://onlinelibrary.wiley.com/doi/full/10.1002/adfm.201705920. This article may be used for non-commercial purposes in accordance with Wiley Terms and Conditions for Use of Self-Archived Versions."

\section{ABSTRACT}

The use of magnetic nanorobots to activate chemotherapeutic prodrugs represents a promising alternative to current chemotherapeutic treatments. Here, a hybrid nanowire (NW) for targeted bioorthogonally driven activation of the latent chemotherapeutic prodrug 5 -fluoro-1-propargyluracil (Pro-5-FU) in in vitro and in vivo cancer models is proposed. The NWs are composed of magnetic iron ( $\mathrm{Fe}$ ) and palladium ( $\mathrm{Pd}$ ), a known bioorthogonal catalyst. In vitro tests with a cancer cell line showed no significant cytotoxic effect by the NWs. In contrast, NWs combined with Pro-5FU lead to a significant reduction of cell viability, similarly to the one induced by its active chemotherapeutic counterpart 5 -fluorouracil $(5-\mathrm{FU})$. The reduction in cell viability is attributed to the catalytic activation of Pro-5-FU into 5-FU. To demonstrate their targeted therapeutic abilities, magnetic fields are used to attract the FePd NWs to a predefined area within a cultured cancer cell population, causing a local Pro-5-FU activation, and subsequent cell death in this region. As a proof of concept, NWs are injected in cancer tumor xenografts. The intraperitoneal injection of Pro-5-FU significantly retards tumour growth without causing significant side effects. This work presents a novel chemotherapeutic approach combining nanorobotics and bioorthogonal activation of prodrugs as an efficient alternative to conventional chemotherapy.

Keywords: bioorthogonal chemistry, cancer therapy, iron-palladium, magnetic actuation, nanowires

\section{INTRODUCTION}

Tremendous research in the field of cancer therapy has opened up new avenues for treating this devastating disease.1 In general, the main goal of cancer treatment is to deliver high doses of antitumor agents to the site of action, while minimizing side effects within the healthy surrounding tissue. 2 A majority of current clinical chemotherapy approaches distribute chemotherapeutic agents throughout the entire body. These treatments lack target specificity and are primarily limited by the reduced target drug concentration and nontargeted tissue distribution. 3 These shortcomings render current treatment methods prone to cause fatal side effects for the patients. 4 Further limitations are the fast deactivation, unfavorable pharmacokinetics, and early clearance of the anticancer drug from the body $5, \underline{6} \mathrm{~A}$ promising path to overcome these problems can be obtained from the progress made in the field of nanobiotechnology, by tailoring advanced nanomaterials to allow for site-specific targeting and on-demand drug activation.z 
One approach to restore the clinical use of promising anticancer drugs, with limited pharmacokinetic profiles, is to transform chemotherapeutic agents into latent prodrugs, which are only medically effective upon catalytic activation. $\underline{8}$ This strategy has led in the past to clinical trials and approvals of conventionally unfavorable anticancer drugs. 9 -11 Initially, the activation of biolabile prodrug systems was dependent on a metabolic activation via enzymes in the organism. However, this approach requires either a change in the metabolic environment of the host by artificially introducing enzymes in the body, or lacks target specificity due to the nonspecific abundance of this enzyme throughout the whole organism.12 A second class of prodrugs, so called the bioorthogonal prodrugs, are inspired by the work of Bertozzi on copper free azide-alkyne cycloaddition and Staudinger ligation.13 Bioorthogonal prodrugs are physiologically stable precursors, which can be activated through biocompatible heterogeneous catalysts by means of bioorthogonal organometallic (BOOM) reactions.14 Novel strategies are currently focused on transition metals as catalysts for BOOM reactions, such as copper (I), ruthenium (II), and rhodium (II). However, only a few known reactions are suitable to work under physiological conditions without causing toxic side effects. 15 - $\underline{17}$

Palladium (Pd), being biocompatible $\underline{18}-\underline{20}$ and exhibiting strong catalytic activity, is a promising transition metal candidate for bioorthogonal reactions.21 Recent research by Weiss et al. demonstrated the spatially defined Pd mediated bioorthogonal activation of 5 -fluoro-1propargyluracil (Pro-5-FU) and $\mathrm{N}$-propargyloxycarbonylgemcitabine prodrugs to their respective anticancer agents 5 -fluorouracil (5-FU) and gemcitabine.22, 23. Although the prodrugs were biologically inert, their activation by $\mathrm{Pd}$ particles embedded in a matrix of glycol-polystyrene resin was sufficient to deprotect the respective propargyl group and restore cytotoxicity and antiproliferative properties. In this context, intratumoral implants loaded with Pd nanoparticles have been successfully used as bioorthogonal catalysts in in vitro cancer models and in vivo zebrafish. However, further in vivo demonstrations, particularly in mammalian model systems, are crucial to assess the actual therapeutic potential with regards to safety and pharmacological profiles. Furthermore, it is desirable to explore other means of device design to administer the transition metal Pd, directly to the tumor site, to render it minimally invasive.

The use of nanorobots for targeted drug delivery has tremendously impacted the field of medical research within the last decades. $24, \underline{25}$ Nanorobots have potential benefits over current state-of-theart medical procedures, such as: (i) targeted drug delivery with increased bioavailability; (ii) reduced human error through computer controlled automated delivery processes; (iii) minimally invasive remote access inside the body; (iv) minimization of undesired side effects; ( $v$ ) early diagnosis; and (vi) constant monitoring. $26, \underline{27}$ Recently, the scientific focus has been on the development of smart micro and nanorobots to transport and deliver pharmaceutical agents to the respective target site. Various manipulation techniques (light, electromagnetic fields, acoustic waves) together with chemical or biological targeting agents further enhanced the target specificity of such devices in vitro and in vivo.28-32 The main challenges of these small-scale robots are still related to the transport of pharmacologically sufficient concentrations of the drug to the target site and the implementation of an efficient remote control release mechanism, exclusively at the region of interest. 33

In this work, we report for the first time, a FePd nanorobot that is capable to target specific sites in the cancer cell population and perform bioorthogonal organometallic activation reactions of the commercially available prodrug Pro-5-FU in vitro. We applied template-assisted electrodeposition for the batch fabrication of heterogeneous FePd nanowires and demonstrated their capability for bioorthogonal activation of Pro-5-FU in an in vitro breast cancer model-the MDA-MB-231 cell line.

INSTITUTO DE INVESTİGAÇÃO E INOVACCÃO EM SAÚDE UNIVERSIDADE DO PORTO

Rua Alfredo Allen, 208 4200-135 Porto Portugal +351220408800 
Using the ferromagnetic properties of Fe within the structure, we successfully showed precise, wireless locomotion and applied this property to demonstrate spatially targeted activation of Pro- 5 FU in vitro. Additionally, we applied the magnetical and bioorthogonal active nanorobots to an in vivo mouse model. We were able to demonstrate for the first time, the therapeutic potential of nanorobots for Pd mediated BOOM reactions of Pro-5-FU, with respect to therapeutic efficiency and safety, by significantly reducing MDA-MB-231 tumor growth in nude mice. Combining nanorobots with bioorthogonal chemistry represents a novel avenue in cancer therapy, potentially minimizing the side effects of conventional chemotherapeutic anticancer drugs and retaining high pharmacological efficiency in the tumor region.

\section{Results and Discussion}

The use of nanorobots in biomedical applications demands an assembly strategy that allows for reliable and controlled batch-fabrication of the device. The FePd nanorobots in this work were fabricated by template-assisted electrodeposition in anodized aluminum oxide (AAO) templates (Figure 12a). Coelectrodeposition of Fe (II) and Pd (II) is a challenging system due to the large differences in standard potential ( $\mathrm{Fe}(\mathrm{II})--0.44 \mathrm{~V} ; \mathrm{Pd}-+0.98 \mathrm{~V}$ ). An electrolyte solution composed of only the two metals initiates a redox reaction, causing oxidation of $\mathrm{Fe}(\mathrm{II})$ to $\mathrm{Fe}$ (III) and precipitation of reduced $\mathrm{Pd}(\mathrm{II})$ as well as $\mathrm{Fe}(\mathrm{OH})_{3}$

$\mathrm{Pd}^{\iota+}+2 \mathrm{Fe}^{2+} \rightarrow \mathrm{Pd}^{0}+2 \mathrm{Fe}^{3+}$

Therefore, the addition of complexing agents is needed in order to bring the standard potentials of both metals closer together and to avoid further redox reactions and precipitation of the constituents in the electrolyte. It has been reported that ammonia sulfate and ammonia citrate are effective stabilizing agents, which prevent ferric hydroxide precipitation and further reduce the noble standard potential of $\mathrm{Pd}$ according to the following reactions 34,35

$$
\begin{aligned}
& \mathrm{PdCl}_{2}+4 \mathrm{NH}_{3} \rightarrow\left[\mathrm{Pd}\left(\mathrm{NH}_{3}\right)_{4}\right] \mathrm{Cl}_{2} \quad E^{0}=0.0 \mathrm{~V} \\
& \mathrm{Fe}^{3+}+5 \mathrm{NH}_{4}^{+}+2 \mathrm{C}_{6} \mathrm{H}_{5} \mathrm{O}_{7}^{3-} \rightarrow\left(\mathrm{NH}_{4}\right)_{5}\left[\mathrm{Fe}\left(\mathrm{C}_{6} \mathrm{H}_{4} \mathrm{O}_{7}\right)_{2}\right]+\mathrm{H}_{2} \\
& E^{0}=-0.014 \mathrm{~V}
\end{aligned}
$$

Subsequently, the electrolyte bath was prepared by forming this tetraamine palladium (II) chloride complex by adding ammonia sulfate at a $\mathrm{pH}$ of 1 until all of the $\mathrm{Pd}$ was dissolved. Afterward, ammonium citrate dibasic was added to allow the introduction of $\mathrm{FeCl}_{3}$ salts. Next, after vigorous stirring for $2 \mathrm{~h}$, we received a clear solution and used ammonium hydroxide to increase the $\mathrm{pH}$ value to 9 . The resulting electrolyte bath (Table $\mathrm{S}_{1}$, Supporting Information) was used for electrodeposition of FePd nanowires at a current density of $-2 \mathrm{~mA} \mathrm{~cm}^{-2}$. Scanning electron microscopy image of the resulting FePd nanowires with an average length of $4 \mu \mathrm{m}$ is shown in Figure $\underline{1} \mathrm{~b}$. Transmission electron microscopy (TEM) electron diffraction analysis in Figure $\underline{1} \mathrm{c}$ confirmed the presence of a polycrystalline alloy of FePd. Interestingly, electrodeposited FePd nanowires (Figure 1 d,e) showed a homogeneous distribution of Fe and Pd across the surface. Here, TEM-energy dispersive X-ray spectroscopy (EDX) analysis revealed the distribution of Fe ( $\approx 50$ at\%) and Pd throughout the nanowire. Annealing the nanowires at $700{ }^{\circ} \mathrm{C}$ induced the segregation of both elements, leading to the formation of Fe- and Pd-rich regions across the nanowire (Figure $\underline{1} f, g$ ).

(1) 
Next, we assessed the biomedical applicability of our fabricated FePd nanowires as a catalyst for the bioorthogonal prodrug activation in vitro. For the purpose of this work, we have chosen MDA-MB231 breast cancer cell line, since breast cancer is a highly heterogeneous disease, which represents the most commonly diagnosed and second leading cause of death in women in the western hemisphere.36 MDA-MB-231 cell line was derived from a metastatic pleural effusion of a breast cancer patient and has been extensively studied for in vitro and in vivo experiments. A scheme of action of the proposed system is represented in Figure 2 a. Upon application of the biochemically inert prodrug Pro-5-FU, no effect on an in vitro breast cancer model (MDA-MB-231) is observed. However, the introduction of the FePd nanorobots is expected to catalyze the bioorthogonal deprotection of Pro- 5 FU by cleaving off the attached propargyl group. The resulting biochemically active 5 -FU anticancer agent will then enter the cancer cell, followed by its conversion to the active metabolite by deoxyuridine monophosphate induced apoptosis. In the following experiment, the colorimetric MTT assay was used for the quantification of metabolic activity that corresponds to cell viability.

Pd is already an established and proven biocompatible material frequently used in implantable medical devices.37 However, the biocompatibility of electrodeposited Fe nanowires is somewhat controversial.38, 39 Hence, before testing the functionality of our FePd nanowires, it was pivotal to determine the biocompatibility of the electrodeposited FePd nanorobots toward cancer and noncancerous cell lines. Figure $\underline{2} b$ presents the results of the colorimetric MTT assay of MDA-MB-231 cells conducted with increasing concentrations of FePd nanowires even up to $200 \mathrm{ppm}$. The results show no significant reduction of cell viability toward increasing FePd concentrations. This effect was also found on human $3 \mathrm{~T}_{3}$ fibroblast cells cocultured with $100 \mathrm{ppm}$ of FePd nanowires. Even after $72 \mathrm{~h}$ no apparent morphological changes have been observed at increased nanowire concentrations (Figure $\mathrm{S}_{1}$, b, Supporting Information), rather ${ }_{3} \mathrm{~T}_{3}$ cells started to interact with the nanowires by either internalization or growing on top of them (Figure S2, Supporting Information). Also, the corresponding cell viability assay did not show any cytotoxic effects up to $200 \mathrm{ppm}$ of FePd (Figure S1c, Supporting Information).

Next, we tested the biochemical activity and inertness of 5-FU and Pro-5-FU, respectively. Three different concentrations of the drug and prodrug were added to MDA-MB-231 breast cancer cells and cell viability was assessed after $72 \mathrm{~h}$ by MTT (Figure $\underline{2} \mathrm{C}$ ). As expected, 5 -FU significantly reduced the cell viability by $50 \%$ at $0.2 \times 10^{-3} \mathrm{~m}$ and $70 \%$ at $1 \times 10^{-3} \mathrm{~m}$, when compared to cells treated with the biochemical inert Pro-5-FU (5-20\% at 0.2 or $\left.1.0 \times 10^{-3} \mathrm{~m}\right)$.

After confirming the biocompatible properties of the FePd nanowires, as well as the biochemical inertness of the Pro-5-FU, we went one step further to assess the potential of FePd as a bioorthogonal catalyst under physiological conditions in a MDA-MB-231 cell culture. In this case, breast cancer cells were cultured with Pro- 5 -FU $\left(0.5 \times 10^{-3} \mathrm{~m}\right)$. After cell attachment, various concentrations of annealed and as electrodeposited (not annealed) FePd nanowires were introduced to the cell culture. Figure $\underline{2} \mathrm{~d}$ shows that an increasing amount of FePd nanowires (annealed/not annealed) led to a significant reduction in cell viability compared to the control experiments. The effective conversion efficiency of $100 \mathrm{ppm}$ FePd nanowires was quantified by high-performance liquid chromatography measurements to be at $67 \%$ after $72 \mathrm{~h}$ of incubation (Figure $\mathrm{S}_{3}$, Supporting Information). In fact, these results proof for the first time that FePd-based nanorobots are capable of inducing the bioorthogonal conversion of Pro-5-FU to 5-FU. 
Next, we tested the efficiency of our system based upon varying amounts of Pro-5-FU at a constant FePd nanowire concentration (100 ppm). Figure ze shows the MTT results of four different Pro- 5 FU concentrations in combination with annealed or not annealed nanowires. Again, the bioorthogonal activation of Pro-5-FU resulted in a significant reduction in cell viability, even at the lowest concentration of $0.5 \times 10^{-3} \mathrm{~m}$. Increasing Pro-5-FU concentrations further reduced the MDA-MB-231 cell viability. Interestingly, upon a concentration of $1.5 \times 10^{-3} \mathrm{~m}$ Pro-5-FU, the annealed FePd nanowires showed a significantly increased efficiency of the Pro-5-FU compared to the aselectrodeposited FePd samples. Considering the redistribution upon annealing of the Fe and Pd on the nanowire (as shown in Figure $1 \mathrm{~g}$ ), we assume that the formation of Pd-rich regions increased the local Pd concentration on the nanowire surface. Consequently, the higher amount of Pd on the surface is expected to increase the conversion efficiency of Pro-5-FU and hence, lead to an augmented pharmacological profile.

After we have confirmed the bioorthogonal capability of FePd nanowires, we tested the application of our system for spatially targeted cancer therapy. Due to the ferromagnetic properties of Fe in the nanowires, wireless magnetic manipulation can be applied for a noninvasive and precise locomotion of the nanorobots to the target site. In order to simulate such a setup, MDA-MB-231 cells were cultured in a Petri-dish and incubated with Pro-5 FU and 100 ppm FePd nanowires (Figure 3 i). Afterward, we placed a magnet under a defined area of the tissue culture dish. After $72 \mathrm{~h}$, we used a LIVE/DEAD staining kit for the cells and imaged different parts of the entire culture. The two fluorescent dyes of the kit, SYTog and propidium iodide, allowed differentiating the alive (green) and dead/compromised cells (red), respectively. On the left site (ii) (region without the magnet), we observed significantly lower amounts of FePd nanowires and most of the cells present in that area showed a green (alive) fluorescence signal. On the other hand, the right site (iii) was heavily populated with FePd nanowires due to the attraction by the applied magnet. Consequently, in that area FePd nanowires could effectively catalyze the bioorthogonal conversion of Pro-5-FU to 5-FU and hence cells in that region were predominantly compromised, as indicated by the red fluorescence signal. Furthermore, we have verified the mobility of FePd nanowires using uniform low-magnitude rotating magnetic fields. Video S1 (Supporting Information) demonstrates the guided locomotion of the FePd nanowires along a square trajectory.

Finally, we validated the in vitro results in an in vivo preclinical mouse model. For this, breast cancer cells (MDA-MB-231) were subcutaneously injected in the right flanks of female nude mice (Figure $4 \mathrm{a}$ ). Tumors were allowed to grow for a week, until reaching a mean average tumor volume of $100 \mathrm{~mm}^{3}$. At this time, mice were randomly distributed in five groups; two of them (group 4 and group 5) received an intratumoral injection of a FePd nanowire suspension $\left(25 \mathrm{mg} \mathrm{kg}^{-1}\right)$ in $100 \mu \mathrm{L}$ phosphate buffered saline (PBS), while the other groups (Groups 1, 2, and 3) were injected with the same volume of PBS. Thereafter, three times a week for a total period of two weeks, mice were intraperitoneally injected with active 5-FU (40 mg kg-1; Group 3) and inactive Pro-5-FU (40 mg kg-1, Groups 2 and 5) (Figure $\mathrm{S}_{4}$, Supporting Information). Groups 1 and 4 received the vehicle alone. Figure $4 \mathrm{~b}$ shows the development of relative tumor volume growth of all five groups. Interestingly, the groups treated with the active drug 5-FU (Group 3) and FePd nanowires in combination with Pro-5-FU (Group 5) showed a significant reduction in tumor growth, when compared to the control mice (PBS only (Group 1); Pro-5-FU only (Group 2); FePd nanowires only (Group 4)). Moreover, tumor growth inhibition of the activated prodrug was comparable to the active chemotherapeutic agent. This result confirms the in vivo activation of the Pro-5-FU by FePd nanowires injected in the tumor. The administered nanowires were observed throughout the experiment in the tumor region of all mice

INSTITUTO DE INVESTİGAÇÃO E INOVACCÃO EM SAÚDE UNIVERSIDADE DO PORTO

Rua Alfredo Allen, 208 4200-135 Porto Portugal +351220 408800 
(Figure $4 \mathrm{C}$ ), which was further confirmed after dissection of the tumor (Figure $4 \mathrm{~d}$ ). Further, histological evaluation of the recovered tumors from mice showed a decrease in vascular invasion in mice treated with FePd nanowires and Pro-5-FU, as well as with only 5-FU (only 1 in 4 mice showed signs of vascular invasion). This result suggests that the biological effect promoted by the active 5 FU and FePd activated Pro-5-FU are comparable (Figure $4 \mathrm{e}$ ). Additionally, biochemical analysis of the blood for liver (Figures S5a and S6, Supporting Information) and kidney (Figure S5b, c, Supporting Information) toxicity markers, such as alanine aminotransferase, urea, and creatinin, respectively, did not show any significant differences between FePd nanowires, Pro-5-FU, and 5-FU groups. The fact that 5 -FU did not show any indications of side effects could mainly be attributed to the short time frame of the experiment, since previous studies reported only significant long-term side effects in vivo.40, 41 The results were also validated histologically, where no differences between the liver and kidney tissue of the animal groups was observed (Figures S6 and S7, Supporting Information). Furthermore, immunohistochemical staining for ki67 (a cellular proliferation marker), showed the same trend as for tumor growth and for vascular invasion, where both groups 5 -FU and FePd nanowires with Pro- $5-\mathrm{FU}$, have a decrease in the percentage of positive (proliferating) cells $(91.25+/-1.25$ vs $81+/-3.317$ (Pro- 5 -FU vs $5-F U)(p=0.0346)$ and $87+/-3$ vs $83^{+/-1.225}$ (FePd nanowires with PBS vs FePd nanowires with Pro-5-FU) (Figure S8, Supporting Information). The FePd nanowires were identified both in the histological sections (Figure S7, Supporting Information) and by TEM analysis (Figure $4 \mathrm{f}-\mathrm{h}$ ). Through TEM analysis, it was possible to confirm that the FePd nanowires were uptaken by the breast cancer cells, but had no influence on their morphology or cell viability (Figure $4 \mathrm{f}, \mathrm{g}$ ). Apart from this, in mice treated with FePd nanowires and Pro-5-FU, the TEM observation again confirmed the cellular uptake of nanowires and further showed a significant increase in the number of dead and apoptotic cells within the tissue (Figure $4 \mathrm{~h}$ ).

\section{Conclusion}

In this work, we combined for the first time a magnetically driven, noncytotoxic nanorobot for the bioorthogonal activation of the chemotherapeutic prodrug-Pro-5-FU-and tested the effect of this system for cancer therapy. The nanowires composed of Fe and Pd entities were synthesized by template-assisted electrodeposition. Biocompatibility tests, both in vitro and in vivo, did not show any significant cytotoxic effects, enabling the use of FePd nanowires for biomedical applications. Next, we demonstrated the bioorthogonal conversion of the latent Pro-5-FU to the active chemotherapeutic $5-\mathrm{FU}$ in the presence of FePd nanowires by initiating significant cell death in a breast cancer in vitro cell model. The magnetic properties of Fe present in the nanowires allowed us to extend our in vitro study to demonstrate targeted cancer therapy. Here, we used magnetic fields to attract FePd nanowires to predefined cancer areas, triggering the local activation of Pro-5-FU and subsequent cell death exclusively in this region. Finally, we transferred the findings of our novel approach to an in vivo mice model of MDA-MB-231 human breast cancer xenografts in nude mice. The FePd nanowires injected in the tumor significantly reduced tumor growth for intraperitoneal administration of Pro-5-FU over a period of two weeks compared to the control groups (only PBS, only Pro-5-FU, only FePd). 


\section{Acknowledgements}

The authors would like to acknowledge the Scientific Center for Optical and Electron Microscopy (ScopeM) of ETH, and the FIRST laboratory for their technical support. The authors would also like to acknowledge to the Histology and Electron Microscopy Service (HEMS) core facility at i3S - Instituto de Investigação e Inovação em Saúde, University of Porto - specially to Rui Fernandes for the TEM analysis. The authors also acknowledge the pathologist Antonio Polónia for the immunohistological classification of mice tumors. This work partially financed by the European Research Council Starting Grant "Magnetoelectric Chemonanorobotics for Chemical and Biomedical Applications (ELECTROCHEMBOTS)," by the ERC Grant agreement no. 336456 .

This work was further supported by the Short-Term Scientific Mission (STSM) Grant from the COST Action MP1407. and the NORTE-01-0145-FEDER-000012 project, financed by Norte Portugal Regional Operational Programme (NORTE 2020), under the PORTUGAL 2020 Partnership Agreement, through the European Regional Development Fund (ERDF). Animal experiments were carried out in accordance with the European Guidelines for the Care and Use of Laboratory Animals, Directive 2010/63/UE and the National Regulation published in 2013 (Decreto-Lei n. ${ }^{\circ} 113 / 2013$ de 7 de Agosto). All experimental procedures were approved by the Ethics Committee of izS.

\section{Conflict of Interest}

The authors declare no conflict of interest.

\section{Supporting Information}

Supporting Information is available from the Wiley Online Library or from the author. 


\section{REFERENCES}

[1] P. Gotwals, S. Cameron, D. Cipolletta, V. Cremasco, A. Crystal, B. Hewes, B. Mueller, S. Quaratino, C. SabatosPeyton, L. Petruzzelli, J. A. Engelman, G. Dranoff, Nat. Rev. Cancer 2017, 17, 286.

[2] C. Widakowich, G. de Castro Jr., E. de Azambuja, P. Dinh, A. Awada, Oncologist 2007, 12, 1443 .

[3] B. A. Chabner, T. G. Roberts Jr., Nat. Rev. Cancer 2005, 5, 65.

[4] V. MacDonald, Can. Vet. J. 2009, 50, 665.

[5] S. Zimmermann, R. Dziadziuszko, S. Peters, Cancer Treat. Rev. 2014, 40, 716.

[6] S. D. Undevia, G. Gomez-Abuin, M. J. Ratain, Nat. Rev. Cancer 2005, 5, 447.

[7] X. J. Liang, C. Chen, Y. Zhao, P. C. Wang, Methods Mol. Biol. 2010, 596, 467.

[8] Y. Singh, M. Palombo, P. J. Sinko, Curr. Med. Chem. 2008, 15, 1802 .

[9] I. Giang, E. L. Boland, G. M. Poon, AAPS J. 2014, 16, 899.

[10] X. Peng, V. Gandhi, Ther. Delivery 2012, 3, 823.

[11] J. Rautio, H. Kumpulainen, T. Heimbach, R. Oliyai, D. Oh, T. Jarvinen, J. Savolainen, Nat. Rev. Drug Discovery $2008,7,255$.

[12] P. Ettmayer, G. L. Amidon, B. Clement, B. Testa, J. Med. Chem. 2004, 47, 2393.

[13] E. M. Sletten, C. R. Bertozzi, Acc. Chem. Res., 2011, 14, 666.

[14] J. T. Weiss, J. C. Dawson, C. Fraser, W. Rybski, C. Torres-Sanchez, M. Bradley, E. E. Patton, N. O. Carragher, A. Unciti-Broceta, J. Med. Chem. 2014, 57, 5395.

[15] C. S. McKay, M. G. Finn, Chem. Biol. 2014, 21, 1075.

[16] T. Volker, F. Dempwolff, P. L. Graumann, E. Meggers, Angew. Chem., Int. Ed. Engl. 2014, 53, 10536.

[17] G. Y. Tonga, Y. D. Jeong, B. Duncan, T. Mizuhara, R. Mout, R. Das, S. T. Kim, Y. C. Yeh, B. Yan, S. Hou, V. M. Rotello, Nat. Chem. 2015, 7, 597.

[18] S. V. Chankeshwara, E. Indrigo, M. Bradley, Curr. Opin. Chem. Biol. 2014, 21, 128.

[19] X. Q. Huang, S. H. Tang, X. L. Mu, Y. Dai, G. X. Chen, Z. Y. Zhou, F. X. Ruan, Z. L. Yang, N. F. Zheng, Nat. Nanotechnol. 2011, 6, 28.

[20] J. Li, J. T. Yu, J. Y. Zhao, J. Wang, S. Q. Zheng, S. X. Lin, L. Chen, M. Y. Yang, S. Jia, X. Y. Zhang, P. R. Chen, Nat. Chem. 2014, 6, 352 .

[21] A. Dumas, P. Couvreur, Chem. Sci. 2015, 6, 2153.

[22] J. T. Weiss, C. Fraser, B. Rubio-Ruiz, S. H. Myers, R. Crispin, J. C. Dawson, V. G. Brunton, E. E. Patton, N. O. Carragher, A. Unciti-Broceta, Front. Chem. 2014, 2, 56.

[23] J. T. Weiss, J. C. Dawson, K. G. Macleod, W. Rybski, C. Fraser, C. Torres-Sanchez, E. E. Patton, M. Bradley, N.

O. Carragher, A. Unciti-Broceta, Nat. Commun. 2014, 5, 3277 .

[24] G. V. da Silva Luz, K. V. G. Barros, F. V. C. de Araújo, G. B. da Silva, P. A. F. da Silva, R. C. I. Condori, L. M. Brasil, Mater. Sci. Eng. A 2016, A6, 167.

[25] R. A. Freitas Jr., J. Nanosci. Nanotechnol. 2006, 6, 2769.

[26] P. Pamies, A. Stoddart, Nat. Mater. 2013, 12, 957.

[27] K. H. Reza, G. Asiwarya, G. Radhika, B. Dipankar, Int. J. Pharm. Sci. Rev. Res. 2011, 10, 60 . 
[28] M. Hoop, F. Mushtaq, C. Hurter, X. Z. Chen, B. J. Nelson, S. Pane, Nanoscale 2016, 8, 12723.

[29] X. Z. Chen, M. Hoop, N. Shamsudhin, T. Huang, B. Ozkale, O. Li, E. Siringil, F. Mushtaq, L. Di Tizio, B. J. Nelson, S. Pane, Adv. Mater. 2017, 29, 1605458.

[30] J. Li, C. Fan, H. Pei, J. Shi, Q. Huang, Adv. Mater. 2013, 25, 4386.

[31] H. Wang, M. Pumera, Chem. Rev. 2015, 115, 8704 .

[32] J. Li, B. E. F. de Ávila, W. Gao, L. Zhang, J. Wang, Sci. Robot. 2017, 2, eaam6431.

[33] K. Park, ACS Nano 2013, 7, 7442.

[34] M. Izaki, in Modern Electroplating, 5th ed. (Eds: M. Schlesinger, M. Paunovic), John Wiley \& Sons, Inc., Hoboken, NJ, USA 2011.

[35] B. Y. Yoo, S. C. Hernandez, B. Koo, Y. Rheem, N. V. Myung, Water. Sci. Technol. 2007, 55, 149.

[36] B. Weigelt, J. L. Peterse, L. J. van't Veer, Nat. Rev. Cancer 2005, 5, 591.

[37] B. Woodward, Platinum Met. Rev. 2012, 56, 213.

[38] Y. P. Ivanov, A. Alfadhel, M. Alnassar, J. E. Perez, M. Vazquez, A. Chuvilin, J. Kosel, Sci. Rep. 2016, 6, 1.

[39] S. Q. Song, G. Bohuslav, A. Capitano, J. Du, K. Taniguchi, Z. H. Cai, L. Sun, J. Appl. Phys. 2012, 111, 056103.

[40] H. I. El-Sayyad, M. F. Ismail, F. M. Shalaby, R. F. Abou-El-Magd, R. L. Gaur, A. Fernando, M. H. Raj, A. Ouhtit, Int. J. Biol. Sci. 2009, 5, 466.

[41] S. A. Thomas, Z. Grami, S. Mehta, K. Patel, Cancer Cell Microenviron. 2016, 3, 1. 
a)
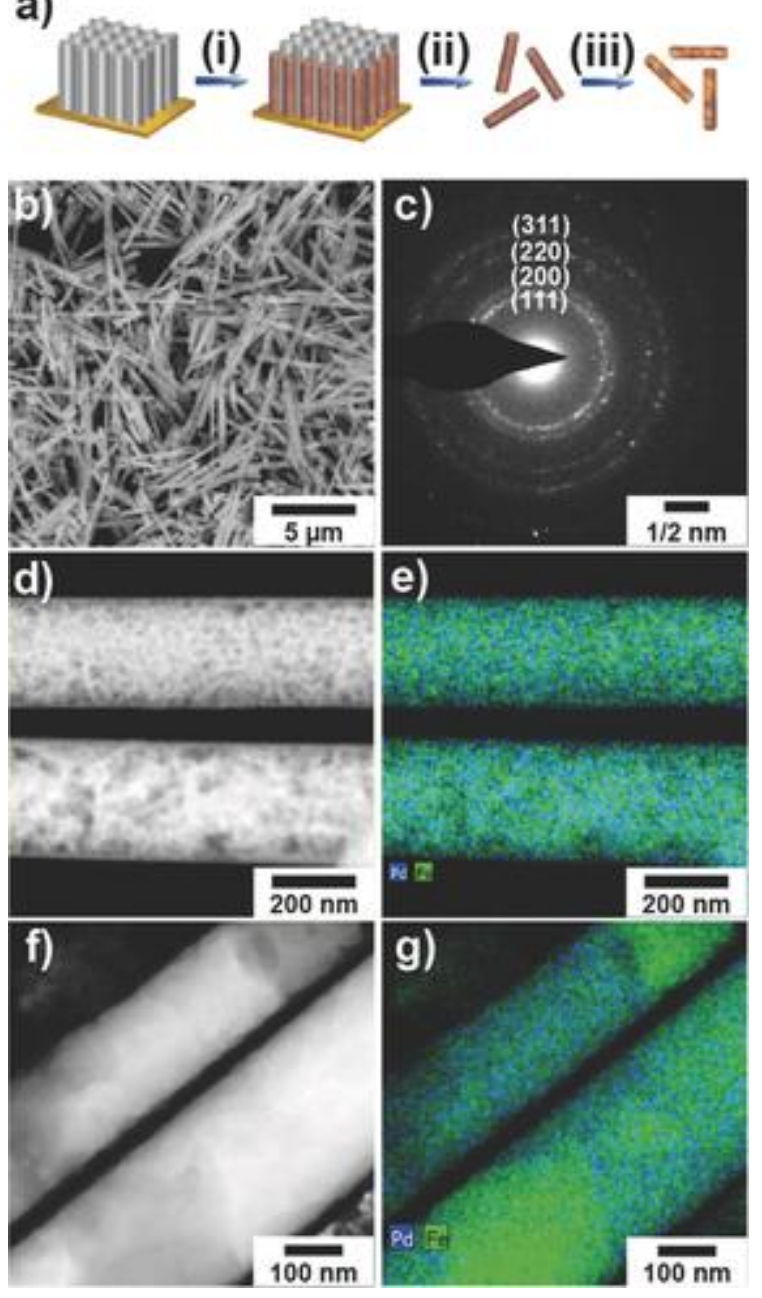

Figure 1. a) Schematic depiction of the electrodeposition of FePd nanowires in AAO (i) followed by the removal of the template via $\mathrm{NaOH}$ etching (ii) and subsequent annealing (iii); b) multiple FePd nanowires after electrodeposition; $\mathrm{c}$ ) TEM diffraction pattern confirming the presence of $\mathrm{FePd}$; $d$,e) scanning transmission electron microscopy (STEM) and TEM - EDX map of Fe (green) and Pd (blue) in as - deposited FePd nanowires; $f, g$ ) STEM and TEM EDX map of Fe (green) and Pd (blue) in annealed FePd nanowires. 
a)
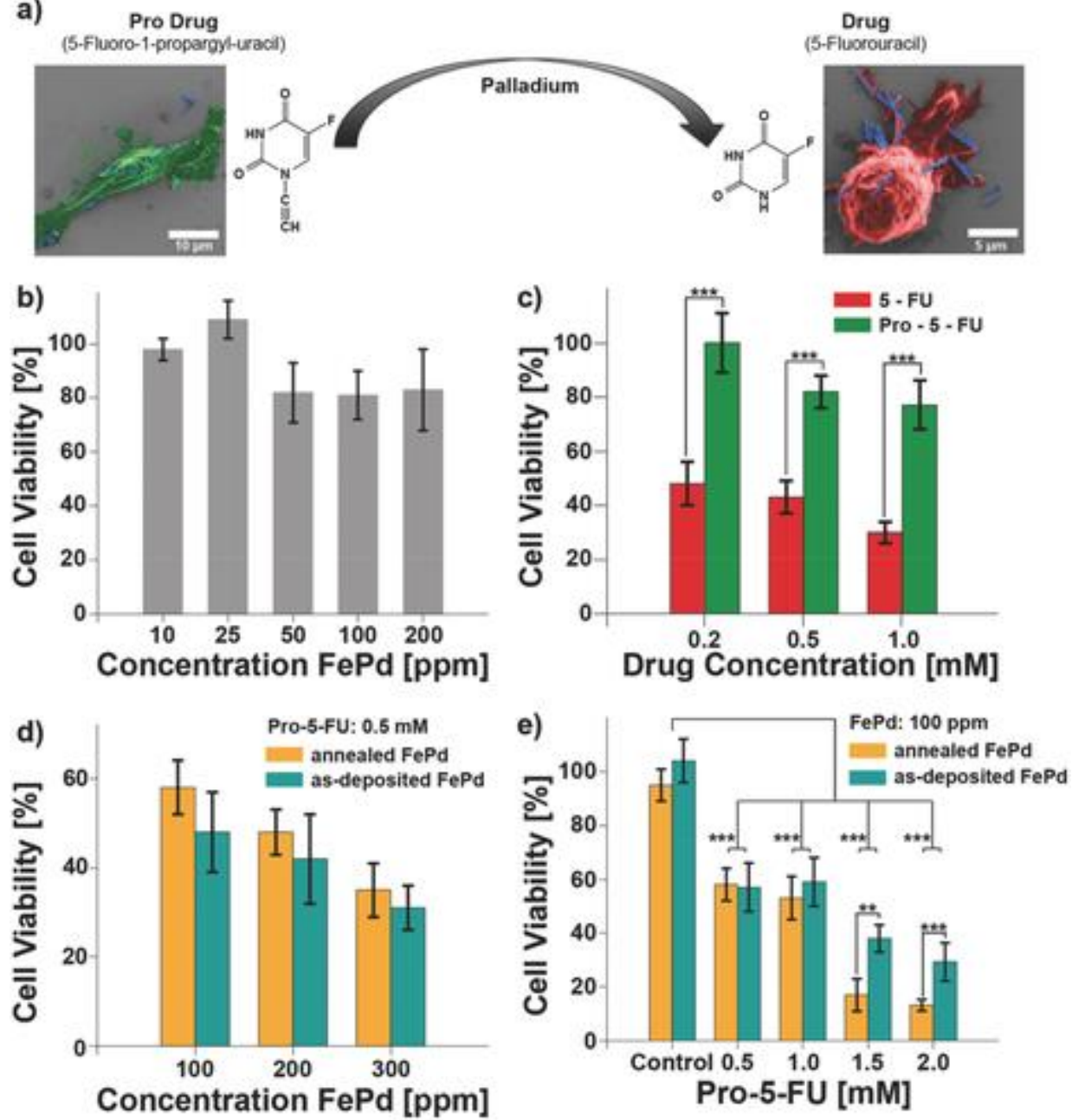

Figure 2. a) Schematic description of the bioorthogonal conversion of the nontoxic Pro-5-FU via the Pd catalyst to the cytotoxic 5-FU; MTT assay results: b) Cell viability results of FePd on MDA-MB-231 cells after $72 \mathrm{~h}$ with different concentration of the nanowires; c) cytotoxicity results on MDA-MB-231 of Pro-5-FU and 5 -FU after $72 \mathrm{~h}$ without FePd nanowires; d) comparison of bioorthogonal activation activity of different FePd nanowire concentrations in the presence of Pro- 5 - $\mathrm{FU}\left(0.5 \times 10^{-3} \mathrm{~m}\right)$ after $72 \mathrm{~h}$; e) comparison of bioorthogonal activation activity with $100 \mathrm{ppm}$ FePd nanowires of different concentrations of Pro-5-FU after $72 \mathrm{~h}_{;} * * p<0.05 ; * * * p<0.01 ; n$ $=8$. 

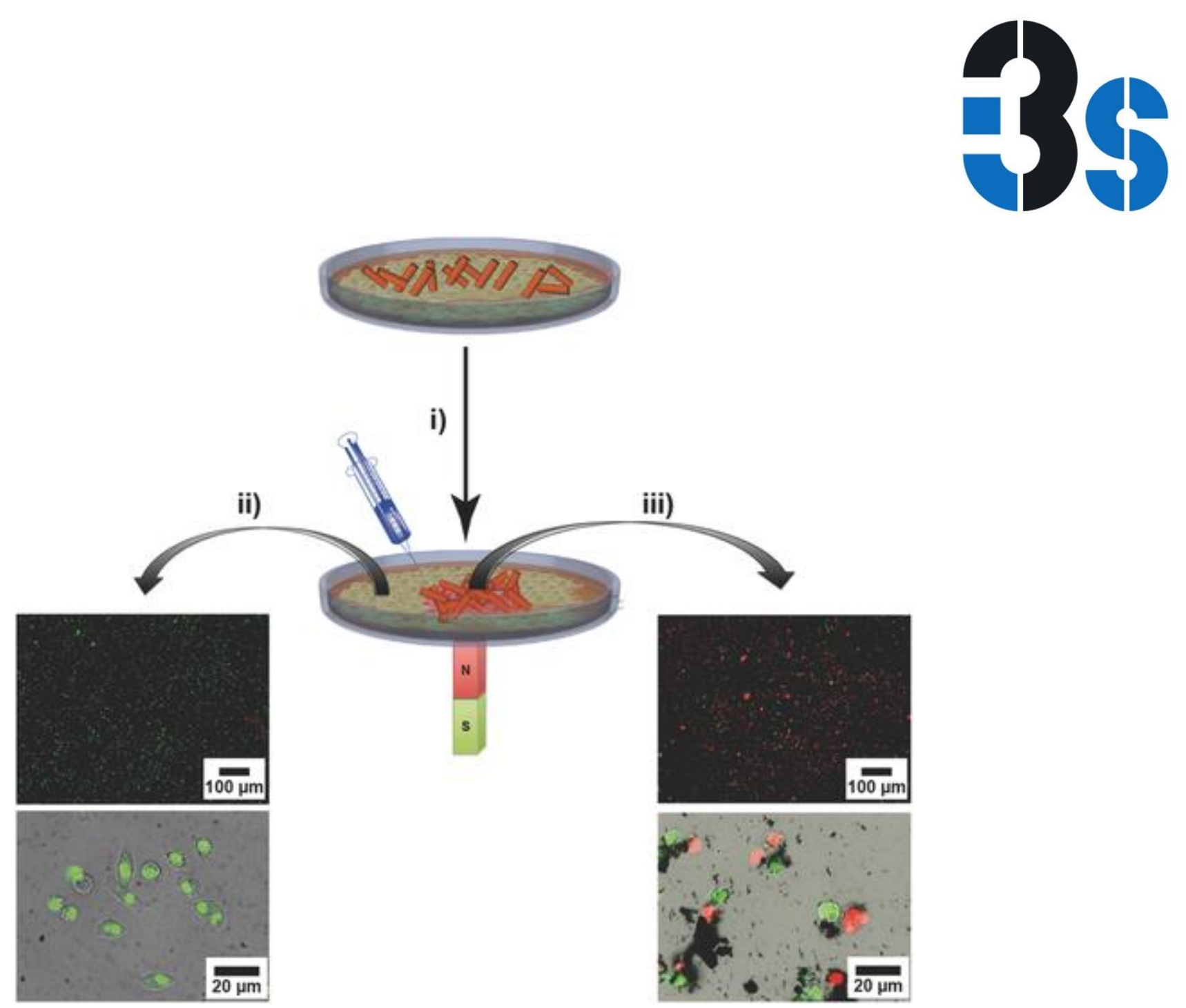

Figure 3. Depiction of the experimental setup to demonstrate in vitro targeted drug activation: i) $100 \mathrm{ppm}$ FePd nanowires are incubated with MDA - MB - 231 cells and $1 \times 10-3 \mathrm{~m}$ Pro - 5 - FU. A magnet is placed under a specific region of the Petri - dish. Results of the LIVE/DEAD staining: ii) Cells away from the magnetic field show green fluorescence indicating viable cells; iii) above the magnet, a high concentration of magnetic FePd nanowires is observed and majority of cells are stained red (compromised cell wall), indicating dead cells due to elevated activation of Pro - 5 - FU in that target region. 
a) ii)

ii) iv)

vi)

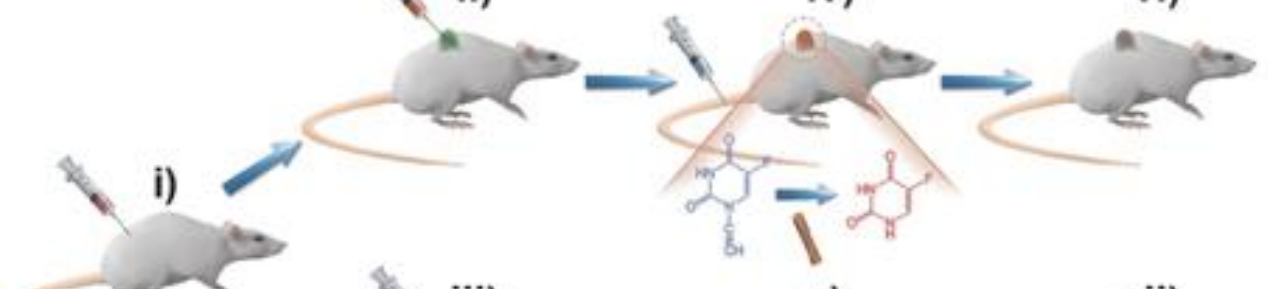

상

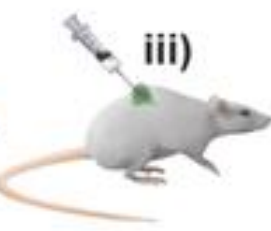

v)

vii)

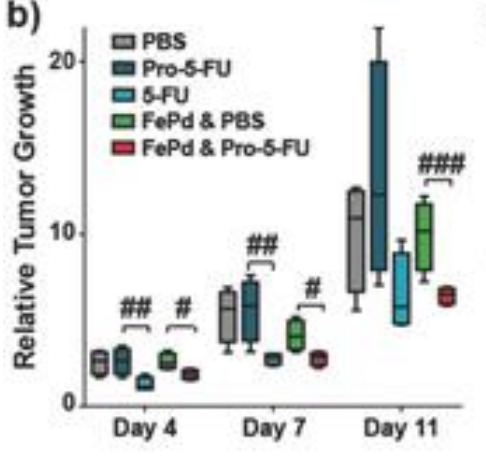

c)

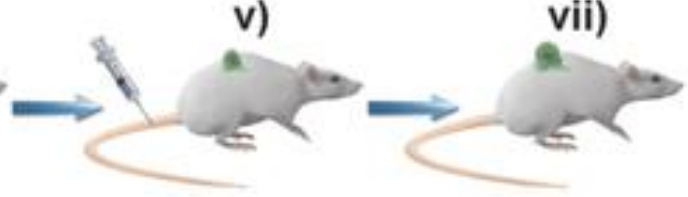

b)
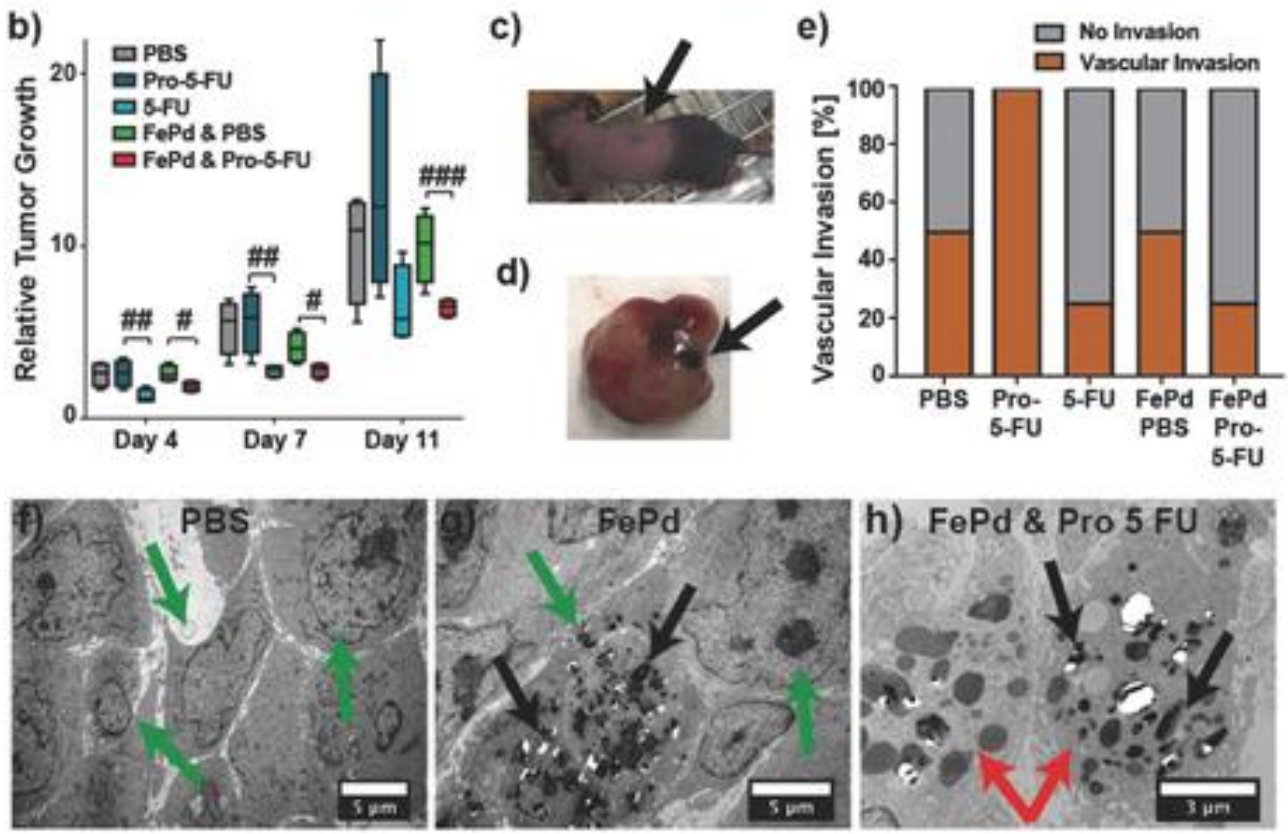

Figure 4. a) Scheme depicting the experimental setup for the in vivo tests: i) MDA - MB - 231 tumor were injected subcutaneously. After the tumor growth either ii) FePd nanowires or iii) vehicle (PBS) were injected into the tumor. Three times a week Pro - 5 - FU was intraperitoneally administered and was either iv) converted by the presence of FePd nanowires to 5 - FU or v) left unaffected. After $14 \mathrm{~d}$, a significant reduction of tumor growth was observed in (vi) compared to (vii). b) Relative tumor volume measured over $11 \mathrm{~d}(\mathrm{n}=4)(\# p<0.04, \# \# p<0.03$; $\# \# \#$ < $<.02)$; c) representative image of a mouse after subcutaneous injection of the nanowires-arrow points to a tumor with nanowires; d) representative image of a tumor after dissection with the FePd nanowires; arrow is indicating the nanowires; e) comparison of the percentage of animals that showed vascular invasion in the tumor region; $f-h$ ) representative TEM image of the tumor from mice treated with f) PBS, g) FePd, and PBS, h) FePd and Pro - 5 - FU, black arrows indicating nanowires, red arrows indicating death/apoptotic cells, green arrows indicating alive cells. 
Supporting Information

for Adv. Funct. Mater., DOI: 10.1002/adfm. 201705920

Mobile Magnetic Nanocatalysts for Bioorthogonal Targeted

\section{Cancer Therapy}

Marcus Hoop, * Ana Sofia Ribeiro, Daniel Rösch, Philipp

Weinand, Nuno Mendes, Fajer Mushtaq, Xiang-Zhong Chen,

Yang Shen, Carlos Franco Pujante, Josep Puigmartí-Luis,

Joana Paredes, Bradley J. Nelson, * Ana Paula Pêgo, * and

Salvador Pané* 
DOI: $10.1002 /$ adfm.201705920

\section{Supplementary Information}

\section{Mobile magnetic nanocatalysts for bioorthogonal targeted cancer therapy}

By Marcus Hoop*, Ana Sofia Ribeiro, Daniel Rösch, Philipp Weinand, Nuno Mendes, Fajer

Mushtaq, Xiang-Zhong Chen, Yang Shen, Carlos Franco Pujante, Josep Puigmarti-Luis,

Joana Paredes, Bradley J. Nelson*, Ana Paula Pêgo*, and Salvador Pané*

\begin{tabular}{ll}
\hline Concentration & \multicolumn{1}{c}{ Chemical } \\
\hline $0.002 \mathrm{M}$ & $\mathrm{PdCl}_{2}$ \\
$0.008 \mathrm{M}$ & $\mathrm{NH}_{4} \mathrm{OH}$ \\
$0.018 \mathrm{M}$ & $\mathrm{FeCl}_{3}{ }^{*} 6 \mathrm{H}_{2} \mathrm{O}$ \\
$0.018 \mathrm{M}$ & $\left(\mathrm{NH}_{4}\right)_{2} \mathrm{SO}_{4}$ \\
$0.018 \mathrm{M}$ & $\begin{array}{l}\text { Ammonium Citrate } \\
\text { Dibasic }\end{array}$ \\
\hline
\end{tabular}

$\mathrm{pH}$ adjusted to $\mathrm{pH} 9$

Table. S1 - Final electrolyte composition for the electrodeposition of FePd nanowires
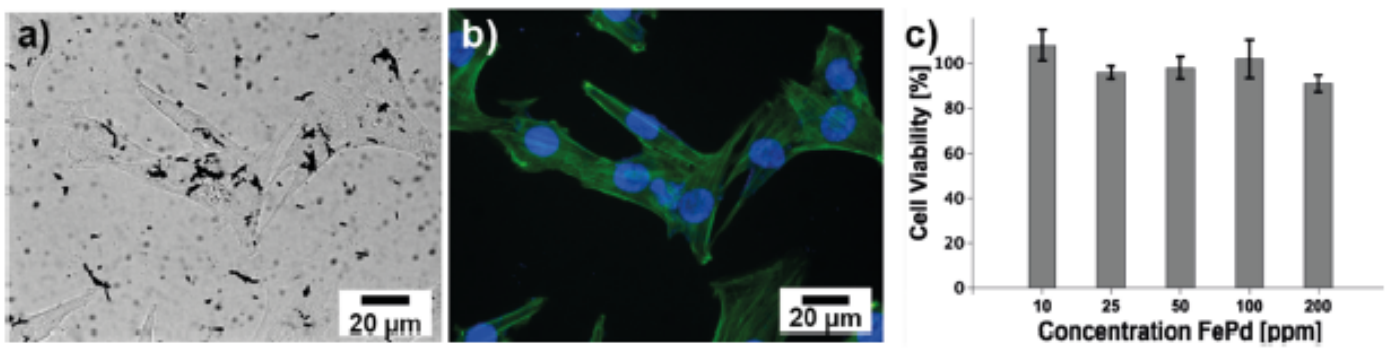

Fig. S1 - Fibroblasts 3T3 cells after 72 hours of incubation with $100 \mathrm{ppm}$ FePd nanowires -

(a) Brightfield image, (b) Fluorescence image: nuclei (blue, Hoechst 33342), actin (green, Phalloidin); (c) Cell viability of various FePd nanowire concentrations on fibroblast 3T3 cells. 

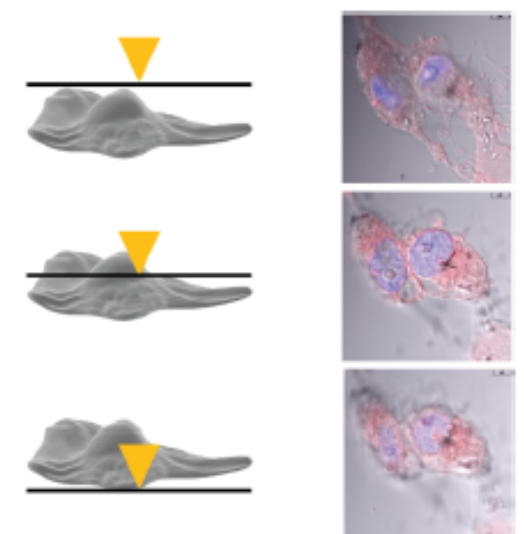

Figure S2 - Confocal microscopy z-stack of FePd nanowires and 3T3 cells.

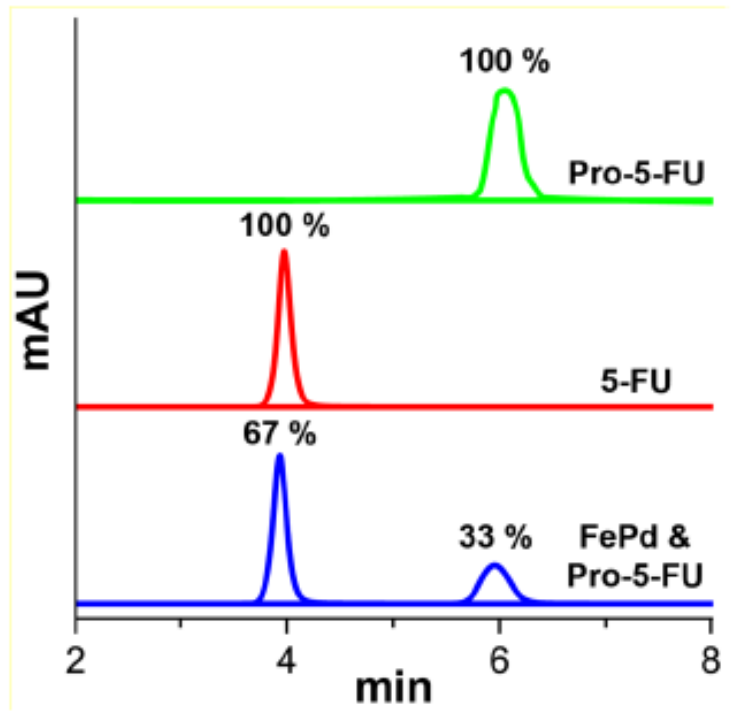

Fig. S3 - HPLC traces of FePd nanowires (100 ppm) mediated bioorthogonal conversion of Pro-5-FU to 5-FU. Conversion efficiency was determined by comparing the area under the respective peaks. 
IN VIVO EXPERIMENTAL DESIGN

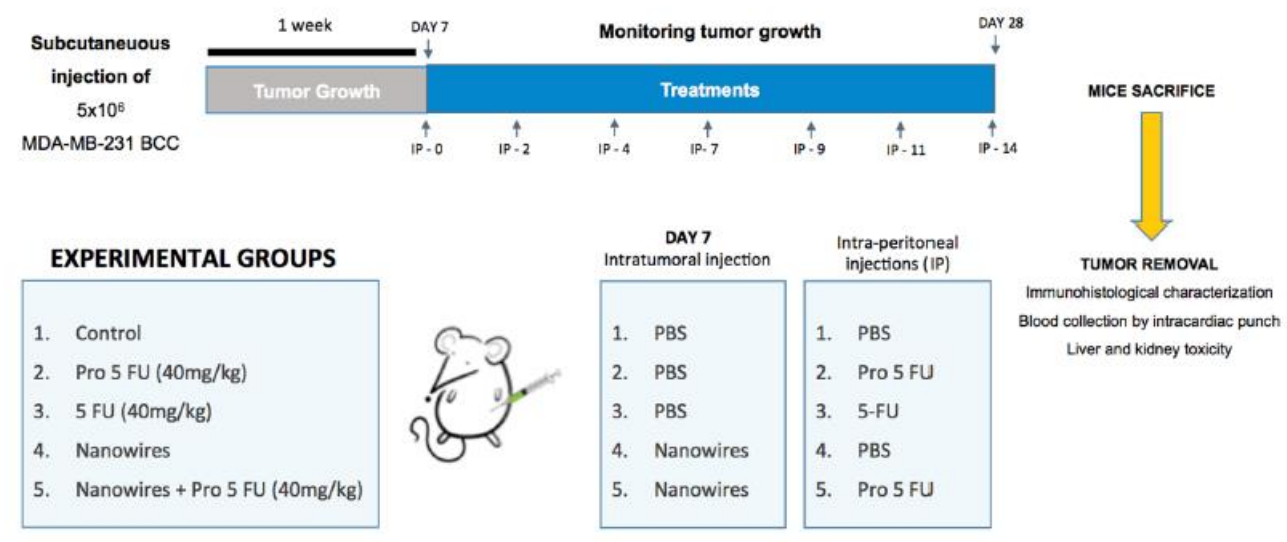

Fig. S4 - Experimental design for the in vivo test

a)

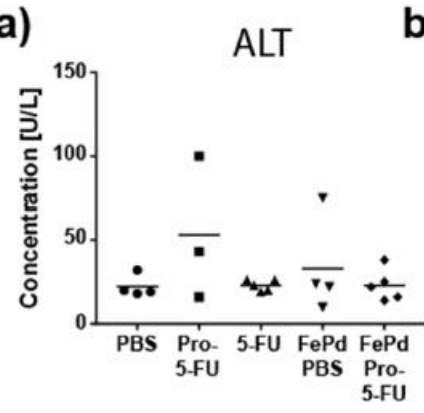

b)

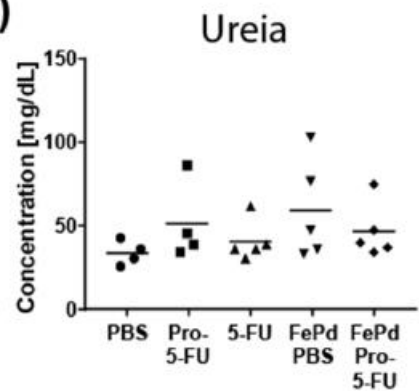

c)

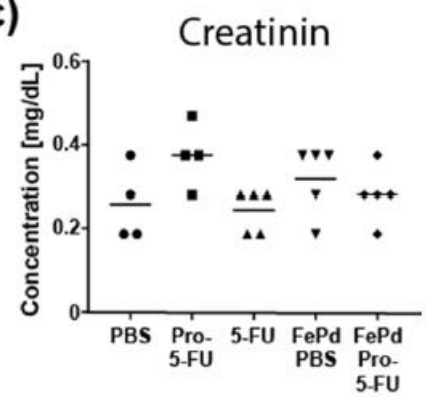

Fig. S5 - Biochemical parameters for liver and kidney function. 

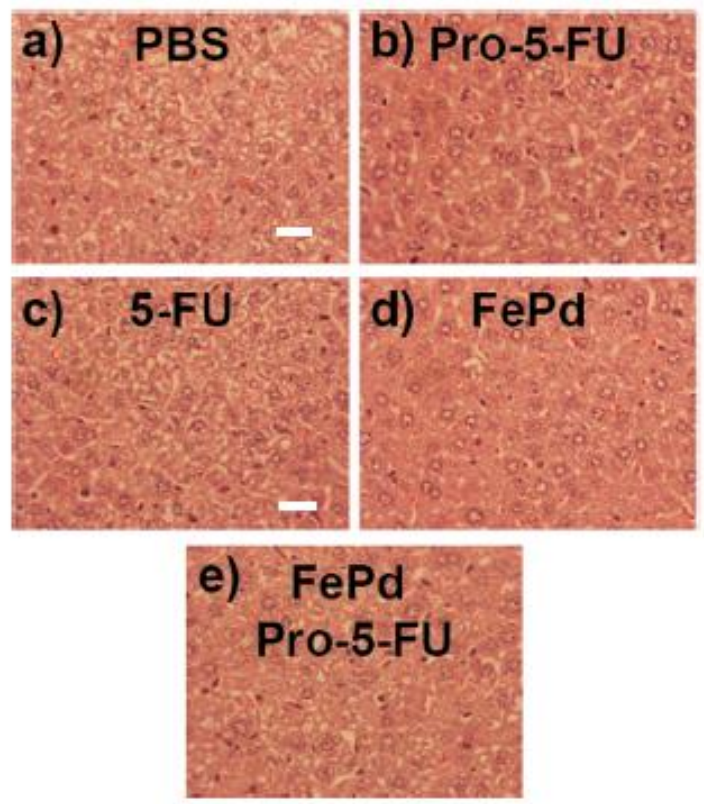

Fig. S6 - Histology (H\&E) of liver sections representative of all mice groups (morphological overview). Scale bar represents $100 \mu \mathrm{m}$.

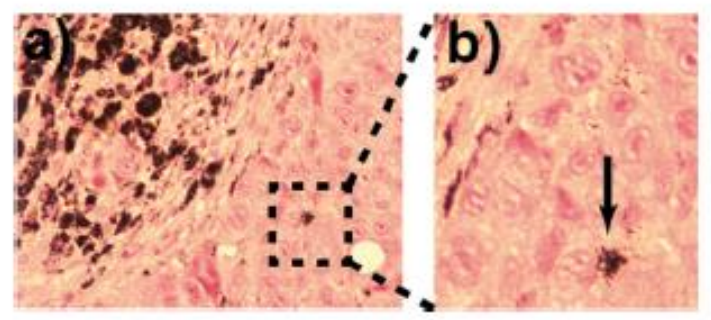

Fig. S7 - Histology (H\&E) of a tumor showing nanowire deposition and uptake by cancer cells. 

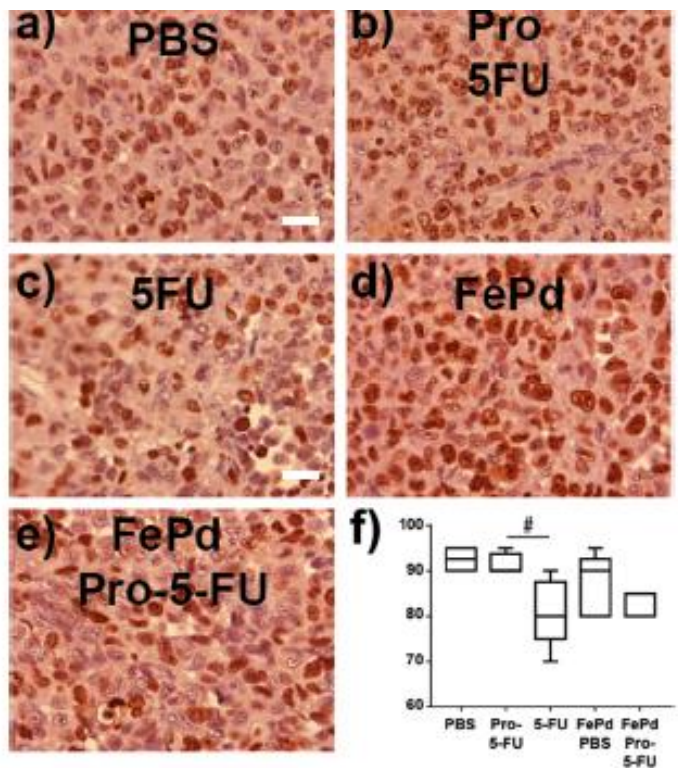

Fig. S8 - Mice tumors - Immunohistochemistry for Ki67. Scale bar represents $100 \mu \mathrm{m}$.

\section{Materials and Methods}

Electrodeposition of Pd and FePd nanowires. Nanowires were deposited into an anodic aluminum oxide (AAO) template (Anodisc 25 from GE) with an average pore diameter of $200 \mathrm{~nm}$. Initially, the templates were sputtered with $100 \mathrm{~nm}$ of $\mathrm{Au}$, followed by the electrodeposition of $1 \mu \mathrm{m}$ Au in each pore (current density $-2 \mathrm{mAcm}-2,40$ $\mathrm{min}$ ) in order to increase the conductivity for the subsequentdeposition. ForFePdnanowires electrodeposition: $0.002 \mathrm{M} \mathrm{PdCl}_{2}$ wasadded to a $0.008 \mathrm{M} \mathrm{NH}_{4} \mathrm{OH}$ solution and the $\mathrm{pH}$ was adjusted to 1 by adding $\mathrm{H}_{2} \mathrm{SO}_{4}$ dropwise, followed by vigorous stirring until the Pd fully dissolved. Afterwards, $0.018 \mathrm{M}\left(\mathrm{NH}_{4}\right)_{2} \mathrm{SO}, 0.018 \mathrm{M}$ ammonium citrate dibasic, and $0.018 \mathrm{M} \mathrm{FeCl}_{3} * 6 \mathrm{H}_{2} \mathrm{O}$ were added and the $\mathrm{pH}$ was to adjusted to 9 with $\mathrm{NH}_{4} \mathrm{OH}$.

Subsequently, FePd nanowires were electrodeposited at $-2 \mathrm{mAcm}-2$ for 2 hours.

After electrodeposition, the Au layer was etched with a commercially available gold etchant (GE-8148, Transene). Then, nanowires were either annealed at $700^{\circ} \mathrm{C}$ in a Rapid Thermal Annealer (RTA) or directly released from the template in a $5 \mathrm{M} \mathrm{NaOH}$ solution, followed by cleaning with water and ethanol.

SEM and TEM imaging of nanowires. Nanowires were dispersed on a silicon chip and imaged via scanning electron microscopy (SEM, Zeiss ULTRA 55). The nanowires were dispersed on to a CU TEM grid (mesh size 400) for transmission electron microscopy (TEM) and energy dispersive x-ray spectroscopy (EDX) (TEM, FEI Talos F200X) analysis.

SEM imaging of cells. Cells were seeded on a Si chip in a 24 tissue culture well plate and incubated at physiological conditions $\left(37^{\circ} \mathrm{C}, 5 \% \mathrm{CO}_{2}\right)$ for $4 \mathrm{~h}$ to let the cells attach to the surface. Afterwards, FePd nanowires (100 ppm) were added to the cell culture and incubated for an additional 72 hours. Then, cells were fixed for $15 \mathrm{~min}$ in $4 \%(\mathrm{w} / \mathrm{v})$ paraformaldehyde. The Si chip with fixed cells was washed 3 times in DI (deionized) water and placed in 1-butyl-3-methylimidazolium tetrafluoroborate for $30 \mathrm{~s}$. Then, the cells were washed in a container with DI water for 30 sec and air dried, before imaging with SEM (Zeiss Ultra).

In vitrocell viability study. Stock solutions of 5 -fluorouracil (5 FU) and 5-fluoro-1-propargyluracil (Pro 5 FU) were prepared in DMSO. For the following experiments, drug and PBS concentrations always contained $1 \%(v / v)$ DMSO. 
in a 96-well plate (Costar ${ }^{\circledR}$ ) 2 × 103 MDA-MB-231 (ATC TCC ${ }^{\circledR}$ CRM-HTB-26 $6^{\text {TM }}$ ) cells in cell media (DMEM-Glutamax, $10 \% \mathrm{w} / \mathrm{V}$ FCS, PenStrep) were seeded and incubated for 4 hours before treatment. Afterwards, the respective amount of nanowires and $5 \mathrm{FU} / \mathrm{Pro} 5 \mathrm{FU}$ were added. After $72 \mathrm{~h}$ of incubation under physiological conditions $\left(37^{\circ} \mathrm{C}, 5 \% \mathrm{CO}_{2}\right)$, the supernatant was replaced by $100 \mu \mathrm{L}$ fresh cell media, supplemented with $12 \mathrm{mM}(3-[4,5-$ Dimethylthiazol-2-yl]-2,5-diphenyltetrazolium bromide (MTT). After $4 \mathrm{~h}$ of incubation, isopropanol (100 $\mu \mathrm{L}$ ) and $\mathrm{HCl}(0.04 \mathrm{M})$ were added to the cells. Absorbance measurements were conducted in a microtiter plate reader (Infnite M200 Pro, Tecan AG, Mannedorf, Switzerland) at $540 \mathrm{~nm}$. All results were normalized to the untreated cells (100\%).

In a 24 -well plate $2 \times 104$ fibroblast ${ }_{3} T_{3}$ cells were seeded and incubated with various amounts of FePd nanowires. After $72 \mathrm{~h}$ either a MTT assay was conducted or cells were fixed with $4 \%$ paraformaldehyde and stained with Hoechst 33342 (Thermofischer) and Phalloidin (ThermoFisher) according to the suppliers protocol. Images were obtained using an inverted optical microscope (Olympus IX-81).

High-performance liquid chromatography (HPLC). HPLC analyses were carried out with a HPLC pump (JASCO PU-2080 Plus), a ChromNAV chromatography data system (JASCO, Tokyo, Japan), and using an ultraviolet detector (JASCO MD-2018 Plus detector) set at $280 \mathrm{~nm}$. The column employed was a reversed phase column YMC-Pack ODS-A $(250 \times 46 \mathrm{~mm})$. The mobile phase used was a mixture of water (A) and acetonitrile with $0.1 \%$ of trifluoro acetic acid (B); A/B = 95:5 to 5:95 in $15 \mathrm{~min}$ and at a constant flow rate of $1 \mathrm{ml} / \mathrm{min}$. The prodrug (Pro-5FU) and drug (5-FU) were dissolved in water / 10\% DMSO and analyzed separately by HPLC. The retention time of Pro-5-FU and $5-\mathrm{FU}$, in the conditions used, were approximately 6 min and $4 \mathrm{~min}$, respectively.

LIVE/DEAD assay for demonstrating targeted drug activation in vitro. 107 MDAMB- 231 cells in cell culture media were seeded in tissue culture polystyrene dishes (diameter $15 \mathrm{~cm}$; Corning) and were incubated overnight to allow for cell adhesion. Then, FePd nanowires (100 ppm) in DMEM were added and supplemented with $2 \mathrm{mM}$ Pro $5 \mathrm{FU}$. The petri dish was placed back into the incubator and a magnet was placed underneath one site of petri dish. 72 hours later, a LIVE/DEADTM BacLightTM viability assay (Life Technology) was conducted, according to the manufacturer's instructions. After $10 \mathrm{~min}$ of incubation the cell viability was assessed with an epi-fluorescence inverted optical microscope (Olympus IX-81) at wavelength of 480/500 nm (SYTO9, green fluorescent nucleic acid stain for viable cells) and 490/635 nm (PI, propidium iodide, a membrane-impermeable fluorescent dye which binds to DNA; marker for dead or inviable cells).

Magnetic actuation. The FePd nanowires were manipulated by a customized magnetic actuation system (MFG100-I, MagnetbotiX AG, Switzerland) in DI water. The magnetic fields were generated by eight opposing coils (3 $\mathrm{mT} ; 3 \mathrm{~Hz}$ ). The nanotubes were dispersed in DI water, placed underneath the coil system and imaged with an integrated inverted optical microscope (Olympus IX-81).

In vivo animal studies. Animal experiments were carried out in accordance with the European Guidelines for the Care and Use of Laboratory Animals, Directive 2010/63/UE and the National Regulation published in 2013 (Decreto-Lei n. ${ }^{\circ} 113 / 2013$ de 7 de Agosto). All experimental procedures were approved by the Ethics Committee of i3S. N:NIH(S)II-nu/nu mice, strain described by Azar et al. in 1980, were produced by i3S/lpatimup, housed, bred and maintained at the i3S Animal House, in a pathogen-free environment under controlled conditions of light and humidity.[1] The Humane Endpoints established were the ones in use at the facility for oncology studies and included: i) any signals of distress, suffering or pain; ii) weight loss greater than $20-25 \%$ of the maximum body mass; iii) anorexia and moribund state; size of the tumor greater than $2000 \mathrm{~mm} 3$. The experiments consisted on the subcutaneous injection in the right flank of female nude mice $(n=25)$, with 6-8 weeks of age, with $5 \times 106$ MDAMB-231 breast cancer cells, using a $25 \mathrm{G}$ needle. One week after injection, when the induced primary tumours reached a median volume of $100 \mathrm{~mm} 3$, mice were randomized into five groups. Group 1, 2 and 3 were injected intratumorally (IT) with PBS, whereas Group 4 and 5 were injected IT with a nanowire suspension in PBS (25 $\mathrm{mg} / \mathrm{kg}$ ). Subsequently, intraperitoneal (IP) injections with PBS (negative control, $100 \mu \mathrm{L} ;$ Group 1 and Group 4), Pro-5-Fu (40 mg/kg, $100 \mu \mathrm{L}$, Group 2 and 5) and 5-FU (positive control, $40 \mathrm{mg} / \mathrm{kg}, 100 \mu \mathrm{L}$, Group 3 ) were performed 3 times per week, for a total period of 2 weeks. Mice ( 5 per group) were weighted, and tumor width and length were measured with calipers, twice a week. Tumor volume was estimated by using the equation, $V=0.5 \times a$

INSTITUTO DE INVESTİGAÇÃO E INOVAÇÃO EM SAÚDE UNIVERSIDADE DO PORTO

Rua Alfredo Allen, 208 4200-135 Porto Portugal $+351220408800$ 
$\times b_{2}$, where $V$ is volume, $a$ is the length of the major axis of the tumor, and $b$ is the length of its minor axis. After the 2 weeks of treatments, mice were euthanized accordingly to the established endpoints. Upon euthanasia, primary tumors were collected to $10 \%$ buffered formalin for immunohistological analysis and to $2 \%$ (w/v) buffered glutaraldehyde for TEM.

Immunohistochemistry. Tumor sections were immunostained with primary monoclonal antibody against Ki-67 (SP6, Neomarkers 60). Antigen unmasking was carried out using 1:100 commercial citrate buffer, pH 6.0 (Vector Laboratories, Burlingame, $\mathrm{CA}$ ) at $98^{\circ} \mathrm{C}$, for 30 minutes. After the antigen retrieval procedure, the slides were washed in PBS and submitted to blockage of endogenous peroxidase activity by incubation of the slides in $3 \%(\mathrm{v} / \mathrm{v}$ ) hydrogen peroxide (Panreac, Barcelona, Spain) in methanol (Sigma-Aldrich). The slides were further incubated with blocking serum (LabVision Corporation kit) for 15 minutes and then incubated with the primary antibody, diluted 1:350, for 1 h at room temperature. After washes, the slides were incubated with the secondary antibody associated with horseradish peroxidase-labeled polymer (DakoCytomation) and immediately revealed with diaminobenzidine. Tissues were then counterstained with Mayer hematoxylin, dehydrated, and covered using a permanent mounting solution (Zymed, San Francisco, CA). The nuclear staining was evaluated by a pathologist and quantified as the percentage of positive cells in the all analysed tumor areas. Then, the mean percentage of Ki-67-stained nuclei was calculated for each experimental group. HE and IHO images were taken under a light microscope (Zeiss Axioskop 2, Germany) connected to a colour video-camera (camera HAL module), which captured the image using the Digital Sight DS-L1 system (Nikon, Japan).

TEM imaging of the nanowires in the tumor samples. Samples from each tumor sized $1-3 \mathrm{~mm} 3$ from the 5 groups ( $\mathrm{N}=22$ samples) were fixed with a mixture of paraformaldehyde $4 \% \mathrm{v} / \mathrm{v}$ with $2.5 \% \mathrm{v} / \mathrm{v}$ buffered glutaraldehyde (Merck, Germany) in o.14 M sodium cacodylate buffer (Merck, Germany). All tumors in groups 4 and 5 presented visible nanowires. After $48 \mathrm{~h}$ of fixation (minimum), samples were treated in $2 \%$ osmium tetroxide (Electron Microscopy Sciences, UK) in cacodylate buffer, followed by new fixation in $1 \%$ (w/v) uranyl acetate overnight. After dehydration in a series of highly concentrated ethanol, samples were embedded in Epon resin (Electron Microscopy Sciences, UK). One micrometer sections and ultrathin sections were made with glass and diamond knifes, respectively. Ultrathin sections with $50 \mathrm{~nm}$ thicknesses were prepared using an Ultramicrotome (RMC PowerTome PC model, USA) with diamond knives (Diatome, USA). Sections were mounted on formvarcoated nickel grids, stained with uranyl acetate and lead citrate (Delta Microscopies, France) and examined by TEM (JEOL JEM 1400 transmission electron microscope; Tokyo, Japan) equipped with a CCD digital camera Orious 1100W (Tokyo, Japan). The procedures for TEM were performed at the HEMS core facility at izS, University of Porto, Portugal.

Statistical analyses. The statistical analyses for the in vivo xenograft assays and for the immunohistochemistry data were performed by Graph Pad Prism version 5.oc software (Graph Pad Software, San Diego, CA), unless otherwise stated. ANOVA test was used considering a confidence interval of $95 \%$. P values less than 0.05 were considered statistically significant.

\section{References}

[1] H. A. Azar, C. T. Hansen, J. Costa, J Natl Cancer Inst 1980, 65, 421. 